\title{
Could plasma based therapies still be considered in selected cases with atypical hemolytic uremic syndrome?
}

\author{
Sare Gülfem Özlü̈ ${ }^{1 \oplus}$, Bora Gülhan ${ }^{2 \oplus}$, Özlem Aydoğ ${ }^{3 \oplus}$, Emine Atayar ${ }^{4 \oplus}$, Ali Delibaş ${ }^{5}$, \\ Gönül Parmaksız ${ }^{6 \oplus}$, Elif Bahat Özdoğan ${ }^{7 \oplus}$, Elif Çomak $^{8 \oplus}$, Mehmet Taşdemir ${ }^{9}$, \\ Banu Acar ${ }^{10 \oplus}$, Zeynep Birsin Özçakar ${ }^{11}$, Rezan Topaloğlu ${ }^{2 \oplus}$, Oğuz Söylemezoğlu ${ }^{12 \ominus}$, \\ Fatih Özaltın²,40
}

${ }^{1}$ Department of Pediatric Nephrology, Ankara Yildirm Beyazıt University Faculty of Medicine, Ankara; ${ }^{2}$ Department of Pediatric Nephrology, Hacettepe University Faculty of Medicine, Ankara; ${ }^{3}$ Department of Pediatric Nephrology, Ondokuz Mayıs University Faculty Of Medicine, Samsun; ${ }^{2}$ Nephrogenetics Laboratory, Department of Pediatric Nephrology, Hacettepe University Faculty of Medicine, Ankara; ${ }^{5}$ Department of Pediatric Nephrology, Mersin University Faculty of Medicine, Mersin; ${ }^{6}$ Department of Pediatric Nephrology, Adana Başkent University Faculty of Medicine, Adana; ${ }^{7}$ Department of Pediatric Nephrology, Karadeniz Technical University Faculty of Medicine, Trabzon; ${ }^{8}$ Department of Pediatric Nephrology, Akdeniz University Faculty of Medicine, Antalya; ${ }^{9}$ Department of Pediatric Nephrology, Koc University Faculty of Medicine, Istanbul; ${ }^{10}$ Department of Pediatric Nephrology and Rheumatology, University of Health Sciences Ankara City Hospital, Ankara; ${ }^{11}$ Department of Pediatric Nephrology, Ankara University Faculty of Medicine, Ankara; ${ }^{12}$ Department of Pediatric Nephrology, Gazi University Faculty of Medicine, Ankara, Turkey.

\begin{abstract}
Background. Atypical hemolytic uremic syndrome (aHUS) occurs due to defective regulation of the alternative complement pathway (ACP) on vascular endothelial cells. Plasma based therapy (PT) was the mainstay of the treatment for aHUS for many years until the introduction of therapies targeting blockage of the complement system. The aim of this study was to evaluate patients with aHUS who had been treated with plasma based therapies alone.
\end{abstract}

Methods. The outcomes of seven genetically confirmed aHUS patients ( 2 girls, 5 males) were evaluated by means of clinical presentation, response to plasma therapy, course of the disease during the follow-up period and last status.

Results. The median age of the patients at admission was 6.7 years (IQR 0.7-7.8). Three patients received plasma exchange therapy and the other four patients were treated with plasma infusions. One patient was lost to follow-up after one year; the median duration of follow-up for other patients was 3.7 years (IQR 2.7-6.5). During the follow up, two patients from our historical records when complement blocking therapies had not been in clinical use yet in Turkey, underwent kidney transplantation. One transplant patient experienced an acute rejection episode without graft loss. The remaining five patients had a glomerular filtration rate of more than $90 \mathrm{ml} / \mathrm{min} . / 1.73 \mathrm{~m}^{2}$ at the last visit.

Conclusion. Although we had a relatively small patient population, our findings indicate that PT might still be considered in selected patients particularly in countries where complement blocking therapies are difficult to reach due to their unavailability or costs that are not covered by the health care systems.

Key words: atypical hemolytic uremic syndrome, treatment, plasma infusion, plasma exchange, outcome.

Atypical hemolytic uremic syndrome (aHUS) is an uncommon disease which is characterized

凶 Sare Gülfem Özlü - Fatih Ozaltin sgozlu@ybu.edu.tr - fozaltin@hacettepe.edu.tr

Received 23rd November 2020, revised 7th April 2021, accepted 4th July 2021. with Coombs negative hemolytic anemia, thrombocytopenia and acute kidney injury. ${ }^{1,2}$ Dysregulation of the alternative complement pathway (ACP) plays a major role in its pathogenesis. Pathogenic variations in genes encoding complement factor $\mathrm{H}$ (CFH), complement factor I (CFI), complement factor B 
(CFB), complement 3 (C3), membrane cofactor protein $(\mathrm{MCP})$ and complement factor $\mathrm{H}$ related (CFHR) proteins1-5 as well as diacyl-glycerol kinase- $\varepsilon$ (DGKE), thrombomodulin (THBD), plasminogen, and autoantibodies against $\mathrm{CFH}$ are identified in approximately $60-70 \%$ of the patients. ${ }^{2-5}$

Plasma based therapy (PT) was the mainstay of the treatment for many years. This therapy provides normal complement proteins through plasma infusion (PI) or removes mutant proteins or autoantibodies through plasma exchange (PE). The efficacy of PT is mainly based on expert consensus, anecdotal reports or retrospective studies. ${ }^{3,6,7}$ Although eculizumab, a monoclonal antibody for terminal complement inhibition, has been suggested as a first line therapy for the management of aHUS in recent years, the main limitations of eculizumab are its cost and unavailability in some countries. Therefore, studies are needed to offer alternative approaches. One of them would be PT and therefore place and efficacy of this approach in aHUS patients at the acute stage of the disease should be revisited especially in countries where resources are limited and access to eculizumab is difficult. ${ }^{3,7}$

In the present study, we aimed to evaluate clinical features, response to treatment and outcome of patients with aHUS associated with genetic abnormalities who had been treated with PT alone.

\section{Material and Methods}

\section{Patients}

In November 2013, the Turkish aHUS registry was established with the participation of 26 pediatric nephrology centers in Turkey to collect information on the demographic, clinical, laboratory and genetic features of pediatric aHUS patients. Management strategies, prognosis and drug safety were also recorded. The registry included a prospective collection of pediatric aHUS patients (i.e. those who are less than 18 years at the time of disease onset) and was updated every 3 months in terms of treatment, complications and outcome. ${ }^{8}$

Diagnosis of aHUS was based on Coombs negative microangiopathic hemolytic anemia, thrombocytopenia and acute renal failure. Hemolytic anemia was defined as a level of hemoglobin $(\mathrm{Hb})$ less than $10 \mathrm{~g} / \mathrm{dl}$ and $\mathrm{a}$ presence of schistocytes on peripheral smear. Thrombocytopenia was defined as a platelet count of less than $150,000 / \mathrm{mm}^{3}$. Patients who have Shiga toxin producing E. coli (STEC) infection or other specific infectious diseases and patients with co-existing diseases or drug-related HUS were excluded from the registry. ADAMTS13 activity was screened in all patients and $10 \%$ and above activity was considered to be normal. Serum creatinine was measured using the Jaffe method and estimated glomerular filtration rate (eGFR) was calculated using the new Schwartz formula. ${ }^{9}$ Proteinuria was defined as $\geq 1+$ by urine dipstick test and/or a spot urine protein/creatinine ratio $\geq 0.5 \mathrm{mg} / \mathrm{mg}$ in children aged 6-24 months and $\geq 0.2 \mathrm{mg} / \mathrm{mg}$ in children older than 24 months of age. ${ }^{10}$ Oliguria was defined as urine output $<0.5 \mathrm{ml} / \mathrm{kg} / \mathrm{h}$ or $<$ $500 \mathrm{ml} /$ day $/ 1.73 \mathrm{~m}^{2}$ after immediate neonatal period. Chronic kidney disease (CKD) was defined and staged according to the guidelines of KDOQI (Kidney Disease Outcomes Quality Initiative.). ${ }^{11}$

Renal remission was defined as having an eGFR $>90 \mathrm{ml} / \mathrm{min} / 1.73 \mathrm{~m}^{2}$. Hematological remission was defined as having a hemoglobin level of more than $10 \mathrm{~g} / \mathrm{dl}$ without hemolysis, a platelet count of more than $150,000 / \mathrm{mm}^{3}$, and a normal lactate dehydrogenase level $(<450 \mathrm{U} / \mathrm{L})$. Complete remission was defined as having both hematological and renal remission. Renal failure with complete hematological recovery was considered as partial remission. The presence of proteinuria and/or hypertension and/or eGFR $\leq 89 \mathrm{ml} / \mathrm{min} . / 1.73 \mathrm{~m}^{2}$ for more than a duration of 3 months was defined as renal sequelae.

\section{Genetic analysis}

For those patients and/or parents who gave informed consent for genetic screening, 
mutational analyses via Sanger sequencing for CFH, CFI, MCP , CFB, C3, DGKE and CHFR 5 were carried out at the Nephrogenetics Laboratory of Hacettepe University. CFHR1-3 deletion was evaluated via multiplex ligation-dependent probe amplification (MLPA) analysis.

Anti-complement factor $\mathrm{H}$ autoantibody was searched using the CFH IgG ELISA Kit $\left(\right.$ Abnova $\left.{ }^{\mathrm{TM}}\right)$, according to the manufacturer's recommendations (detection limit 0.6 AU/mL).

The Institutional Ethics Committee of Hacettepe University approved the study on May 2011 (FON10/03-22). Written informed consent was obtained from the parents of each patient.

\section{Statistical analysis}

Data were analyzed by using SPSS v.21 (SPSS Inc. Chicago, II, USA). Demographics and clinical data were evaluated with descriptive statistical analysis methods. The mean, median, standard deviation and interquartile range (IQR) were calculated for the numeric variables.

\section{Results}

\section{Patient characteristics}

Seven patients (5 males, 2 females) with a defined underlying genetic abnormality were included in the study (Table I). Five patients had variations in complement regulatory genes [CFB $(\mathrm{n}=2), C F H(\mathrm{n}=1), C D 46(\mathrm{MCP})(\mathrm{n}=1), C 3$ $(\mathrm{n}=1)]$, one patient had a DGKE variation and one patient had anti-CFH antibody associated with a homozygous CFHR1-3 deletion. The median age was 6.7 years (IQR 0.7-7.8). Except for patients $\# 2$, \#5 and \#6, the remaining were diagnosed before the availability of eculizumab in Turkey. None of the patients had a family history of aHUS. Three had parental consanguinity. Diarrhea was not present before the onset of the symptoms. At the time of diagnosis, eGFR was less than $90 \mathrm{ml} / \mathrm{min} . / 1.73 \mathrm{~m}^{2}$ in all patients. Two patients with $C F B$ variation and one with $M C P$ variation had a normal urine output. Patient \#7 (with anti-CFH antibody/CFHR1-3 deletion) had neurological involvement characterized by seizures during follow-up.

All patients had hypocomplementemia and all but patient \#2 (with CFB variation) had varying degrees of proteinuria and hypertension at admission. Demographic variables, clinical and laboratory features, genetic results are summarized in Table I.

\section{Treatment}

PE or PI was started on the day of diagnosis in all patients (Table I). Patient \#2 was diagnosed after eculizumab approval in Turkey; he was given eculizumab at the time of diagnosis but due to severe anaphylaxis the treatment had to be continued with PE. Overall, patients \#2 (with $C F B$ variation), patient \#3 (with $C F H$ variation) and patient \#4 (with $M C P$ variation) underwent PE with each session of 40-60 ml/ $\mathrm{kg}$ plasma and four patients received PIs (10-20 $\mathrm{ml} / \mathrm{kg} /$ day); two received 7 and two received 8 infusions. Peritoneal dialysis (PD) was started in patient \#6 (with DGKE variation) and patient \#7 (with CFH antibody/CFHR1-3 deletion) and hemodialysis was started in patient \#3 (with $\mathrm{CFH}$ variation). Antihypertensive drugs were administered to all patients (Table I).

\section{Outcome}

Patient \#5 (with C3 variation) was lost to followup after one year however at the time of the last visit, she had an eGFR of $158 \mathrm{ml} / \mathrm{min} . / 1.73 \mathrm{~m}^{2}$. Median follow up duration of the other six patients was 3.7 years (IQR 2.72-6.47). All patients were in hematological remission at the time of discharge. Patient \#3 (with CFH variation) was discharged with hemodialysis and patient \#7 (with anti-CFH antibody/CFHR1-3 deletion) was followed up on PD. Patient \#3 underwent kidney transplantation from a cadaveric donor two years after diagnosis. On the fifth year of kidney transplantation, she suffered from antibody mediated rejection due to incompliance to immunosuppressive medications. She was treated with plasmapheresis and intravenous immune globulin to treat this rejection episode 


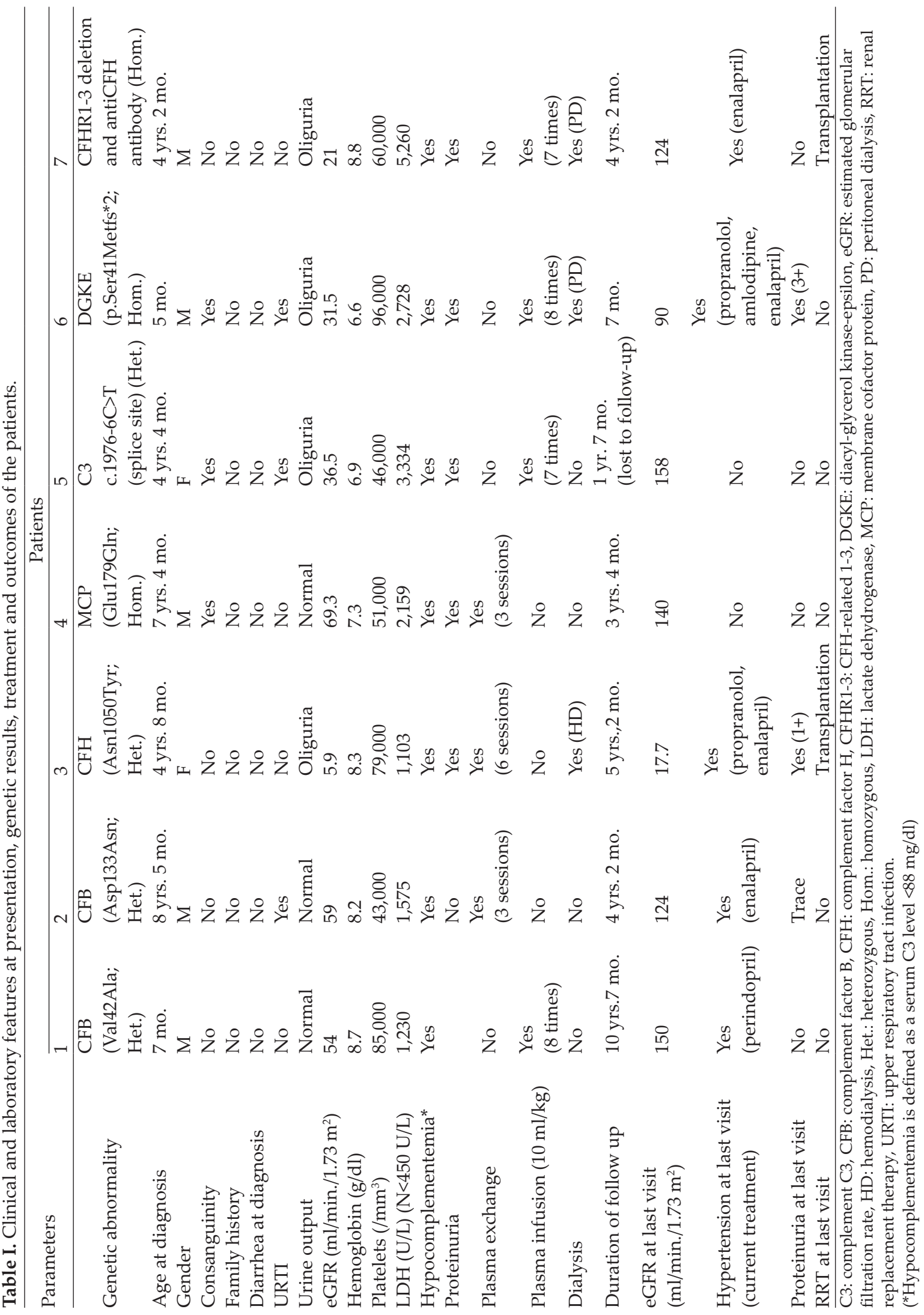


and then was followed up with conservative therapies for CKD. Patient \#7 (with anti-CFH antibody and CFHR1-3 deletion) underwent kidney transplantation seven years after diagnosis and he is in remission for two years. All but patient \#3 had an eGFR $>90 \mathrm{ml}$ / min./1.73 $\mathrm{m}^{2}$. All but patients \# 4 and \#5 (with $M C P$ and $C 3$ variation, respectively) received antihypertensive treatment. Proteinuria was detected in patients \# 2, 3 and 6 (with CFB, CFH and DGKE variation, respectively). All patients were in hematological remission and never experienced aHUS episode again during the follow-up duration. Outcome of the patients are summarized in Table I.

\section{Discussion}

Plasma based therapies had been used for aHUS for approximately 40 years and their efficacies were demonstrated in thrombotic thrombocytopenic purpura. ${ }^{12}$ Recently, it has been suggested that response to PT was in part related to the genetic background of the patient and the most favorable response to PT were reported in patients with anti-CFH antibody and MCP mutations both in short term and long term. ${ }^{13,14}$ Although short term results were acceptable in patients with $C F H$ variations, the risk of end stage renal disease (ESRD) or death was reported as $70-80 \%$ at the end of one year. ${ }^{14}$ Patients with $C F B$ and CFI variations were reported to be poor responders in these studies. ${ }^{13,14}$ After the introduction of complement blocking therapies, there was a sudden change in management that had been in use for 40 years. ${ }^{15,16}$ Complement blocking therapies are effective but also do bring significant risks that could be life-threatening including severe infections and allergic reactions. In addition, these drugs remain one of the most expensive drugs world-wide and there is no certain knowledge about how long they should be administered. There are still difficulties to reach these drugs or reimbursement in the healthcare systems in some countries. Therefore, alternative approaches are certainly needed and research on these approaches should be encouraged. This would open a new avenue into individualized treatment in patients with aHUS. In this context, we aimed to evaluate the outcome of seven patients diagnosed with aHUS who had been treated with plasma therapy alone in the present study.

In our study, one patient with CFHR1-3 deletion/ anti CFH antibody and one with $\mathrm{CFH}$ variation developed ESRD. Both of them were diagnosed with aHUS before eculizumab was approved in Turkey. The patient with CFHR1-3 deletion/ anti-CFH antibody was diagnosed with ESRD soon after the diagnosis and was followed up on PD, underwent kidney transplantation seven years after the first admission. Previous reports have suggested a favorable outcome with a 75\% remission rate in patients with $\mathrm{CFH}$ antibodies who were treated with PT and additional immunosuppression. ${ }^{13,14}$ Recently, it has been reported that outcomes for patients with antiCFH antibodies were marginally inferior to those without antibodies. ${ }^{17}$ Gurjar et al. ${ }^{18}$ have reported that some patients with CFHR1-3 deletion do not have anti-CFH antibodies which led them to suggest that homozygous CFHR1-3 deletion may also contribute to aHUS antibodyindependent mechanisms. Our patient had a worse clinical course than expected which led us to speculate that the co-existence of CFHR1/3 deletion and anti-CFH antibody might have had an additional negative effect on the outcome of the disease. As to the patient with $C F H$ variation with poor prognosis she never responded to $\mathrm{PE}$ and soon after the disease onset she developed ESRD and underwent kidney transplantation two years after diagnosis. Although PEs have been reported to be effective even in patients with severe renal impairment, the overall prognosis of $C F H$ variations is not favorable..$^{18-20}$ Davin et al. ${ }^{21}$ reported that progression to ESRD was associated with an elevated plasma creatinine level at presentation. In our patient with CFH variation, unfavorable prognosis despite duly and intensive PEs could be related to significantly impaired renal functions at admission. Therefore, we think that in both of these patients, complement blocking therapy 
(i.e. eculizumab) would also have been ineffective due to very low GFR at presentation even if we had had an opportunity to use it. This observation would confirm once again the fact that sustained low GFR at presentation should be considered a poor long-term prognostic factor in patients with aHUS.

It has been reported that DGKE-HUS patients present with a slowly progressing proteinuric nephropathy and $80 \%$ of the patients did not have ESRD at the end of 10 years. ${ }^{22}$ In that study, 29 out of 35 aHUS patients were treated with immunosuppression, eculizumab, or PTs at any time of disease course. Sixteen patients were treated with PT and acute improvement was attributed to this management in 10 of them. The authors suggested that the link between DGKE deficiency and the complement cascade was not yet clearly described so therapies targeting complement cascade might not be of benefit. ${ }^{22}$ As reported in most of the patients with DGKE-HUS in the literature, our patient with DGKE variation was diagnosed in the first year of his life. ${ }^{4,22,23}$ At the time of diagnosis, he had oliguria, hypertension and massive proteinuria. He received PIs and underwent PD for 15 days. At his last follow up visit, 7 months after diagnosis he was hypertensive and had 3+ proteinuria and a eGFR of $90 \mathrm{ml} / \mathrm{min} . / 1.73 \mathrm{~m}^{2}$.

CFB variations account for $1-4 \%$ of aHUS cases and have been reported to have a poor prognosis. ${ }^{14}$ In contrast to this report, two patients with $C F B$ variation in our study had favorable outcomes. One of them (patient \#2) was diagnosed after eculizumab approval in Turkey so he was given eculizumab. Because of severe anaphylaxis that developed during the first infusion, we had to switch treatment to PE. The other patient with $C F B$ variation (patient \#1) received only PIs. Both patients had favorable long-term outcomes. Given the fact that $C F B$ variation is a rare cause of aHUS, a clear genotype-phenotype correlation is not possible however it is also plausible that there might be additional modifying factors that would affect the prognosis in CFB-related aHUS.
C3 pathogenic variations that lead to aHUS are also rare and the prognosis in this group is poor with a reported rate of $60 \%$ for ESRD or death at the end of 1 year. ${ }^{14,24}$ In a recent report from Japan, the authors reported that $C 3$ variations were the most frequent genetic abnormality with a rate of $31 \%$ in their cohort. They also reported a remission rate of $92 \%$ on the last follow up visit in these patients. ${ }^{25}$ In line with these findings, our patient with $C 3$ variation also exhibited a favorable outcome with complete remission through $\mathrm{PT}$ alone.

$M C P$ variations are related to a good prognosis despite frequent relapses. ${ }^{13,14,26-28}$ Our patient was treated with three sessions of PEs, he was discharged with renal and hematological remission and he was in complete remission at the last visit. Interestingly, Caprioli et al. ${ }^{26}$ have reported a remission rate of $91 \%$ in those patients with $M C P$ variations who were treated by plasma and $100 \%$ who were not and they have concluded that this could be attributed to the fact of $\mathrm{MCP}$, which is a membrane-bound protein. We think that management should be individualized in patients with $M C P$ variations given the fact that some patients may manifest extensive microvascular thrombosis and severe hypertension and numerous cases would benefit from PTs.

In 2012, soon after approval by FDA, eculizumab was also available in Turkey. It is a promising life-long therapy for aHUS and the 2016 consensus report suggests that firstline and early treatment is effective and safe in children with aHUS for renal recovery. ${ }^{2}$ However, debates still exist for its lifelong administration. It is one of the most expensive drugs in the world; yearly treatment cost per adult patient based on the dosing regimen of administration is reported to be $€ 327.600 .{ }^{29}$ There is no published data regarding its cost in children however as of today its yearly cost for a $30 \mathrm{~kg}$ patient has been estimated as $€ 323.000$ in Turkey. Moreover, the risk of development of severe meningococcal infection, possibility of immune mediated drug reactions and severe anaphylaxis as developed in one of our patients 
cannot be underestimated.30,31 Therefore, the efficacy of alternative approaches in aHUS associated with specific complement variations should be explored. In this aspect, our study is of importance in terms of revisiting ancient and ancillary treatment options.

In conclusion, short- and long-term management of patients with aHUS should be tailored individually considering the patient's clinical course, underlying genetic abnormality and current conditions of healthcare systems of countries. We also believe that in countries where eculizumab is difficult to attain or resources are limited; PTs should still be a reasonable alternative in aHUS patients. Although this is a small-sized study, our results indicate that in selected cases plasma based therapies should still be kept in mind for the management of aHUS and more research on the efficacy of these approaches should be promoted.

\section{Author contribution}

Study conception and design: OS, FÖ; data collection: SGÖ, AD, GP, BÇA, ÖA, EÇ, ZBÖ, $\mathrm{EBÖ}, \mathrm{MT}$; analysis and interpretation of the results: FÖ, BG, SGÖ, ZBÖ, EA; draft manuscript preparation: $\mathrm{SGÖ}, \mathrm{BG}$; critically revision of the manuscript: FÖ, OS, RT. All authors reviewed the results and approved the final version of the manuscript.

\section{Ethical approval}

The Institutional Ethics committee of Hacettepe University approved the study on May 2011 (FON10/03-22).

\section{Source of funding}

This study was supported by the Hacettepe University Scientific Research and Development Office (Grant. No: 010A101009).

\section{Conflict of interest}

The authors declare no conflict of interest.

\section{REFERENCES}

1. Ruggenenti P, Noris M, Remuzzi G. Thrombotic microangiopathy, hemolytic uremic syndrome, and thrombotic thrombocytopenic purpura. Kidney Int 2001; 60: 831-846. https://doi.org/10.1046/j.15231755.2001.060003831.x

2. Loirat C, Fakhouri F, Ariceta G, et al; HUS International. An international consensus approach to the management of atypical hemolytic uremic syndrome in children. Pediatr Nephrol 2016; 31: 1539. https://doi.org/10.1007/s00467-015-3076-8

3. Kavanagh D, Goodship TH, Richards A. Atypical hemolytic uremic syndrome. Semin Nephrol 2013; 33:508-530. https://doi.org/10.1016/j. semnephrol.2013.08.003

4. Ozaltin F, Li B, Rauhauser A, et al. DGKE variants cause a glomerular microangiopathy that mimics membranoproliferative GN. J Am Soc Nephrol 2013; 24: 377-384. https://doi.org/10.1681/ASN.2012090903

5. Bu F, Maga T, Meyer NC, et al. Comprehensive genetic analysis of complement and coagulation genes in atypical hemolytic uremic syndrome. J Am Soc Nephrol 2014; 25: 55-64. https://doi.org/10.1681/ ASN.2013050453

6. Ariceta G, Besbas N, Johnson S, et al; European Paediatric Study Group for HUS. Guideline for the investigation and initial therapy of diarrheanegative hemolytic uremic syndrome. Pediatr Nephrol 2009; 24: 687-696. https://doi.org/10.1007/ s00467-008-0964-1

7. Loirat C, Garnier A, Sellier-Leclerc AL, Kwon T. Plasmatherapy in atypical hemolytic uremic syndrome. Semin Thromb Hemost 2010; 36: 673-681. https://doi.org/10.1055/s-0030-1262890

8. Besbas N, Gulhan B, Soylemezoglu O, et al. Turkish pediatric atypical hemolytic uremic syndrome registry: initial analysis of 146 patients. BMC Nephrol 2017; 18: 6. https://doi.org/10.1186/s12882016-0420-6

9. Schwartz GJ, Muñoz A, Schneider MF, et al. New equations to estimate GFR in children with CKD. J Am Soc Nephrol 2009; 20: 629-637. https://doi. org/10.1681/ASN.2008030287

10. Hogg RJ, Portman RJ, Milliner D, Lemley KV, Eddy $\mathrm{A}$, Ingelfinger J. Evaluation and management of proteinuria and nephrotic syndrome in children: recommendations from a pediatric nephrology panel established at the National Kidney Foundation conference on proteinuria, albuminuria, risk, assessment, detection, and elimination (PARADE). Pediatrics 2000; 105: 1242-1249. https://doi. org/10.1542/peds.105.6.1242 
11. Inker LA, Astor $\mathrm{BC}$, Fox $\mathrm{CH}$, et al. KDOQI US commentary on the 2012 KDIGO clinical practice guideline for the evaluation and management of CKD. Am J Kidney Dis 2014; 63:713-735. https://doi. org/10.1053/j.ajkd.2014.01.416

12. Bukowski RM, King JW, Hewlett JS. Plasmapheresis in the treatment of thrombotic thrombocytopenic purpura. Blood 1977; 50: 413-417. https://doi. org/10.1182/blood.V50.3.413.413

13. Noris M, Caprioli J, Bresin E, et al. Relative role of genetic complement abnormalities in sporadic and familial aHUS and their impact on clinical phenotype. Clin J Am Soc Nephrol 2010; 5: 18441859. https://doi.org/10.2215/CJN.02210310

14. Waters AM, Licht C. aHUS caused by complement dysregulation: new therapies on the horizon. Pediatr Nephrol 2011; 26: 41-57. https://doi.org/10.1007/ s00467-010-1556-4

15. Gruppo RA, Rother RP. Eculizumab for congenital atypical hemolytic-uremic syndrome. N Engl J Med 2009; 360: 544-546. https://doi.org/10.1056/ NEJMc0809959

16. Nürnberger J, Philipp T, Witzke O, et al. Eculizumab for atypical hemolytic-uremic syndrome $\mathrm{N}$ Engl J Med 2009; 360: 542-544. https://doi.org/10.1056/ NEJMc0808527

17. Schaefer F, Ardissino G, Ariceta G, et al; Global aHUS Registry. Clinical and genetic predictors of atypical hemolytic uremic syndrome phenotype and outcome. Kidney Int 2018; 94: 408-418. https://doi. org/10.1016/j.kint.2018.02.029

18. Gurjar BS, Manikanta Sriharsha T, Bhasym A, et al. Characterization of genetic predisposition and autoantibody profile in atypical haemolytic-uraemic syndrome. Immunology 2018; 154: 663-672. https:// doi.org/10.1111/imm.12916

19. Sellier-Leclerc AL, Fremeaux-Bacchi V, DragonDurey MA, et al; French Society of Pediatric Nephrology. Differential impact of complement mutations on clinical characteristics in atypical hemolytic uremic syndrome. J Am Soc Nephrol 2007; 18: 2392-2400. https://doi.org/10.1681/ ASN.2006080811

20. Fremeaux-Bacchi V, Fakhouri F, Garnier A, et al. Genetics and outcome of atypical hemolytic uremic syndrome: a nationwide French series comparing children and adults. Clin J Am Soc Nephrol 2013; 8: 554-562. https://doi.org/10.2215/CJN.04760512

21. Davin JC, Olie KH, Verlaak R, et al. Complement factor $\mathrm{H}$-associated atypical hemolytic uremic syndrome in monozygotic twins: concordant presentation, discordant response to treatment. Am J Kidney Dis 2006; 47: e27-e30. https://doi. org/10.1053/j.ajkd.2005.11.007
22. Lemaire $\mathrm{M}$, Frémeaux-Bacchi $\mathrm{V}$, Schaefer $\mathrm{F}$, et al. Recessive mutations in DGKE cause atypical hemolytic-uremic syndrome. Nat Genet 2013; 45: 531-536. https://doi.org/10.1038/ng.2590

23. Azukaitis K, Simkova E, Majid MA, et al. The phenotypic spectrum of nephropathies associated with mutations in diacylglycerol kinase $\varepsilon$. J Am Soc Nephrol 2017; 28: 3066-3075. https://doi.org/10.1681/ ASN.2017010031

24. Loirat C, Saland J, Bitzan M. Management of hemolytic uremic syndrome. Presse Med 2012; 41(3 Pt 2): e115-e135. https://doi.org/10.1016/j. lpm.2011.11.013

25. Fujisawa M, Kato H, Yoshida $Y$, et al. Clinical characteristics and genetic backgrounds of Japanese patients with atypical hemolytic uremic syndrome. Clin Exp Nephrol 2018; 22: 1088-1099. https://doi. org/10.1007/s10157-018-1549-3

26. Caprioli J, Noris M, Brioschi S, et al; International Registry of Recurrent and Familial HUS/TTP. Genetics of HUS: the impact of $\mathrm{MCP}, \mathrm{CFH}$, and IF mutations on clinical presentation, response to treatment, and outcome. Blood 2006; 108: 1267-1279. https://doi.org/10.1182/blood-2005-10-007252

27. Reid VL, Mullan A, Erwig LP. Rapid recovery of membrane cofactor protein (MCP; CD46) associated atypical haemolytic uraemic syndrome with plasma exchange. BMJ Case Rep 2013; 2013: bcr2013200980. https://doi.org/10.1136/bcr-2013-200980

28. Marini SC, Gomes M, Guilherme R, et al. Atypical hemolytic-uremic syndrome: recurrent phenotypic expression of a patient with MCP gene mutation combined with risk haplotypes. Blood Coagul Fibrinolysis 2019; 30: 68-70. https://doi.org/10.1097/ MBC.0000000000000793

29. National Institute for Health and Care Excellence. National Institute for Health and Care Excellence guidance: eculizumab for treating atypical haemolytic uraemic syndrome. London: NICE, 2015. Available at: https://www.nice.org.uk/ guidance/ hst1/chapter/7-Recommendations-for-furtherresearch (Accessed on November 15th, 2020).

30. Ardissino G, Testa S, Possenti I, et al. Discontinuation of eculizumab maintenance treatment for atypical hemolytic uremic syndrome: a report of 10 cases. Am J Kidney Dis 2014; 64: 633-637. https://doi. org/10.1053/j.ajkd.2014.01.434

31. Wijnsma KL, Duineveld C, Volokhina EB, van den Heuvel LP, van de Kar NCAJ, Wetzels JFM. Safety and effectiveness of restrictive eculizumab treatment in atypical haemolytic uremic syndrome. Nephrol Dial Transplant 2018; 33: 635-645. https:// doi.org/10.1093/ndt/gfx196 